\title{
Anomalous scaling in an age-dependent branching model
}

\author{
Stephanie Keller-Schmidt, ${ }^{1}$ Murat Tuğrul, ${ }^{2}$ Víctor M. Eguíluz, ${ }^{3}$ Emilio Hernández-García, ${ }^{3}$ and Konstantin Klemm ${ }^{1,4,5,6}$ \\ ${ }^{1}$ Bioinformatics, Institute of Computer Science, University Leipzig, Härtelstr. 16-18, 04107 Leipzig, Germany \\ ${ }^{2}$ IST Austria, Am Campus 1, 3400 Klosterneuburg, Austria \\ ${ }^{3}$ IFISC (CSIC-UIB), Instituto de Física Interdisciplinar y Sistemas Complejos, E-07122 Palma de Mallorca, Spain \\ ${ }^{4}$ Bioinformatics and Computational Biology, University of Vienna, Währingerstraße 29, 1090 Vienna, Austria \\ ${ }^{5}$ Theoretical Chemistry, University of Vienna, Währingerstraße 17, 1090 Vienna, Austria \\ ${ }^{6}$ School of Science and Technology, Nazarbayev University, Kabanbay Batyr Ave. 53, 010000 Astana, Kazakhstan
}

(Received 4 October 2013; revised manuscript received 23 May 2014; published 2 February 2015)

\begin{abstract}
We introduce a one-parametric family of tree growth models, in which branching probabilities decrease with branch age $\tau$ as $\tau^{-\alpha}$. Depending on the exponent $\alpha$, the scaling of tree depth with tree size $n$ displays a transition between the logarithmic scaling of random trees and an algebraic growth. At the transition $(\alpha=1)$ tree depth grows as $(\log n)^{2}$. This anomalous scaling is in good agreement with the trend observed in evolution of biological species, thus providing a theoretical support for age-dependent speciation and associating it to the occurrence of a critical point.
\end{abstract}

DOI: 10.1103/PhysRevE.91.022803

PACS number(s): 89.75.Da, 87.23.Kg, 89.75.Fb, 89.75.Hc

\section{INTRODUCTION}

Tree structures appear in a variety of contexts ranging from river networks [1] and blood vessels [2] to directed polymers $[3,4]$ or computer file systems [5-7]. Evolutionary histories and genealogies are naturally represented as trees. Each branching point represents an ancestral relationship in a population or an event of diversification on sets of languages [8], species [9-13], or sociocultural innovations [14]. Based on genetic information, modern computational biology has inferred thousands of trees, so-called phylogenies [15], depicting the evolutionary relationships between sets of species, from bacteria to mammals [16]. The shapes of the collected phylogenies and of related evolutionary trees [17] share statistical properties not observed in trees generated by standard branching models [18-20]. It has been a longstanding and fundamental question in evolutionary biology to identify which processes accurately describe the observed tree shapes and thus may serve as models of biological evolution [21,22].

A suitable starting point and null hypothesis is the equal rate Markov (ERM) process, which assumes that species speciate at a constant homogeneous rate, independently of previous events and of other species present. More specifically, starting from a single tip (root), at each discrete time step a tip $i$ is chosen uniformly at random and two new tips are attached to $i$, increasing the number of tips by 1 . The procedure has a direct interpretation for macroevolution as a sequence of speciation events, where the chosen species $i$ is the latest common ancestor of two new species. The resulting topology of the growing tree, which is equivalent to the one produced by the Yule model [23], tends to generate compact and nearly balanced tree shapes. Balance refers to an even distribution of the number of nodes in the subtrees arising from the branches created at each speciation point. However, when comparing with the shape of large collections of observed phylogenetic trees available nowadays (e.g., Refs. $[16,17,24]$ ), the ERM hypothesis can be rejected, as most real phylogenetic trees are significantly less balanced than those generated by the ERM and Yule models [11,21,22,24].

\section{TREE SHAPE AND DEPTH}

Several indices for imbalance measurement have been proposed, used, and compared; see Ref. [24-27] for detailed discussion. Here we study how the depth [28] of a tree with $n$ tips,

$$
d=n^{-1} \sum_{i=1}^{n} d_{i}
$$

scales with $n$. For each tip $i, d_{i}$ denotes the number of edges separating $i$ from the root. The role of the depth in capturing tree imbalance is apparent from the two extreme cases. For the (fully balanced) complete binary tree, $d=\log _{2} n$ since all $n=2^{k}$ tips are at distance $k$ from root. On the other extreme of full imbalance, a comb (or pectinate) tree has $n$ tips attached to a path of $n-1$ nodes starting at the root. Here $n d=\sum_{i=1}^{n} d_{i}=1+2+\cdots+(n-2)+2(n-1)$, resulting in asymptotically linear scaling $d \sim n$. For the ERM model, the small random imbalances introduced in the process are not enough to affect the dominant scaling behavior of the balanced tree and one finds $\langle d\rangle \sim \log n$ (the average is over realizations of the random process). This logarithmic scaling is a robust outcome related to the exponential growth of tips occurring in time for virtually any model of growing supercritical trees [18], as far as branches split independently and without memory, or if these correlations and memory are sufficiently short-ranged. Therefore we denote the logarithmic scaling of depth with tree size as normal. Deviating scaling is called anomalous.

We have calculated $[11,12]$ the depth $d$ for all trees (and subtrees) in the phylogenetic databases TreeBASE (containing species phylogenies [16]) and PANDIT (protein phylogenies [17]). The result in Fig. 1(a) suggests that the average depth grows with the number of tips as

$$
\langle d\rangle \sim(\log n)^{2}
$$

in good approximation. Although alternative scaling laws have been proposed [11,29], the $(\log n)^{2}$ form is more accurate for large tree sizes $[12,30]$. Similar behavior is observed in virus phylogenies where the scaling for individual phylogenies 



FIG. 1. (Color online) Scaling of tree depth with size $n$. (a) Trees from databases TreeBASE and PANDIT. Trees have been binned by size such that each bin contains at least $s$ trees using $s=1000$ for PANDIT and $s=200$ for TreeBASE. Least squares fits (dashed lines) of the form $\sqrt{d}=y=a x+b$ with $x=\ln n$ yield $a=0.657 \pm$ $0.008, b=0.53 \pm 0.03$ with correlation coefficient $r=0.9986$ for TreeBASE; and $a=0.771 \pm 0.006, b=0.48 \pm 0.02, r=0.9990$ for PANDIT. (b) Depth from age and AB models. Fits analogous to the above yield $a=0.654 \pm 0.002, b=0.54 \pm 0.02, r=0.99995$ for the age model with $\alpha=1.0, \Delta t=1 ; a=0.556 \pm 0.003, b=$ $0.72 \pm 0.02, r=0.998478$ for the AB model; $a=0.822 \pm 0.006$, $b=0.20 \pm 0.05, r=0.99959$ for the age model with $\alpha=1.0, \Delta t=$ $1 / n$. Vertical error bars indicate (average \pm standard deviation) $)^{1 / 2}$, in (a) for the average $[d]$ taken over trees inside a bin and in (b) for the mean depth $\langle d\rangle$ estimated by 100 independent realizations at each given size $n$. Horizontal error bars in panel (a) give average \pm Std. Dev. over the tree sizes inside each bin.

was reported to follow the behavior $(\log n)^{\gamma}$ with $\gamma$ varying from 1 to 3 [24]. The important point is the departure from the $\log n$ scaling of the ERM class. Thus strong correlations are important in the evolutionary processes represented in the phylogenetic databases.

\section{STATISTICAL ENSEMBLES OF TREES}

A direct approach to capturing the imbalance of phylogenetic trees is by defining a probability $\pi(l \mid n)$ of placing exactly $l$ out of $n$ given tips in one of the two subtrees. This stochastic splitting is first applied at the root and then iterated at the roots of its two subtrees, at their subtrees' roots and so forth, until arriving at the tips. A statistical ensemble of trees is constructed by considering all possible binary trees up to a given size, and assigning a probability to each of them as just the product of the splitting probabilities of all the inner nodes.

Choosing uniform probabilities independently of $l$, $\pi_{\mathrm{ERM}}(l \mid n)=1 /(n-1), 1 \leqslant l \leqslant n-1$, leads to the ERM. Aldous' branching (AB) model [31] is the specific choice $\pi_{\mathrm{AB}}(l \mid n) \propto l^{-1}(n-l)^{-1}$, placing more probability mass on the less balanced splits close to $l=1$ and $l=n-1$. The AB model is a specific case of the one-parametric (with parameter $\beta$ ) family of beta-splitting models [31]. Statistical quantities computed from the $\mathrm{AB}$ ensemble (parameter value $\beta=-1$ ) have been identified as giving a good fit to real data [21,22,32]. The expected depth scales as $(\log n)^{2}$ [31]. It is interesting to note that the $\mathrm{AB}$ case $\beta=-1$ is precisely the critical point separating two qualitatively distinct scaling behaviors in the general beta-splitting model: standard logarithmic scaling for $\beta>-1$, and power-law scaling $\langle d\rangle \sim n^{-\beta-1}$ for $\beta<-1$ [31].

The AB model, beta-splitting, and other models [33] introduced to account for tree imbalance, however, assign probabilities to tree shapes in a way which is not based on any evolutionary mechanism. While they can statistically reproduce features of the trees in the databases, this does not hint at any biological explanation of these features, as Ref. [21] remarks.

\section{THE AGE MODEL}

\section{A. The model and its depth}

We introduce the age model, which describes the growth of a binary tree by iterative stochastic addition of tips, one at each time step. Each tip $i$ is assigned an age $\tau_{i}(t)$ being the time that passed from the birth of the tip, $t_{i}$, to the present time $t$, i.e., $\tau_{i}(t)=t-t_{i}$. At time $t=0$ the tree consists of a single tip (the root), labeled with the index $i=1$, representing an ancestral species. The growth proceeds by iterating the following three steps: (i) A tip $i$ is chosen with probability $p_{i}(t)$ inversely proportional to a power of its age

$$
p_{i}(t)=\frac{\tau_{i}^{-\alpha}}{c_{\alpha}(t)},
$$

where the normalization constant $c_{\alpha}(t)$ is chosen such that probabilities from all tips sum up to 1; (ii) a new branch $j$ is split from $i$ with creation time $t_{j}=t$ while tip $i$ remains; (iii) time $t$ is increased by $\Delta t$ and the process resumes at (i). Each branching represents a new species evolutionarily splitting from the original one. This is coherent with a scenario of peripatric speciation, in which a small part of an ancestral population becomes isolated and starts an independent evolution process, whereas the main part of the population continues its previous dynamics. We focus on $\Delta t=1$ first and discuss the case of $\Delta t=1 / n$ later, in Sec. IV C. There we also regard a variation that treats both new descendants of the split branch as new, starting at age zero. In terms of biological evolution, the symmetric splitting is allopatric speciation. 


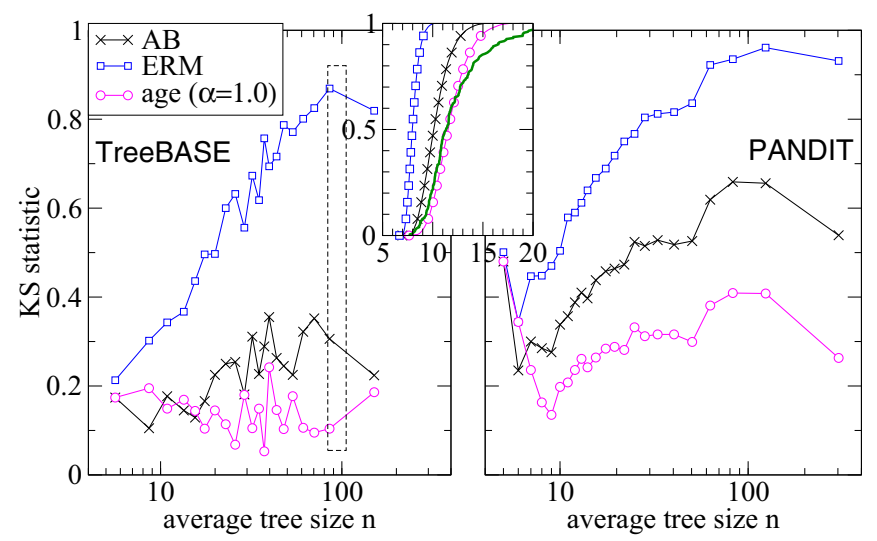

FIG. 2. (Color online) Comparison between data and models (AB, ERM and age) by distributions of depth. The large panels show the maximum deviation (Kolmogorov-Smirnov statistic) between the cumulative distribution of depth in each model and the real trees. Subsets $\mathcal{T}$ of the databases are chosen the same as the bins in Fig. 1(a). See the main text for further details. The inset shows, for one subset of TreeBase (trees of size $76 \leqslant n \leqslant 102$ ), the cumulative distributions of the real trees (thick curve without symbols) and the three models, leading to the KS statistic values marked by the dashed rectangle.

This defines a family of models parameterized by $\alpha$. We note that $c_{\alpha}(n)$ (and $p_{i}(n)$ ) depends only on the values of $n$ and $\alpha$ (and $i$ ). These quantities are independent of the details of the previous branching history, which is a stochastic process. The ERM model, in which at each step one tip is uniformly (independently of its age) chosen for speciation, is recovered for $\alpha=0$. Negative $\alpha$ enhances branching probability of the oldest tips, so that trees more balanced than random ones are expected. We will see that for sufficiently large positive $\alpha$ (in fact $\alpha \geqslant 1$ ) the excess branching probability given to the youngest tips strongly breaks balance and modifies the ERM logarithmic depth scaling.

Figure 1(b) shows the dependence of mean depth on tree size for the age model for several choices of the exponent $\alpha$. At $\alpha=1.0$, we obtain $\langle d\rangle \sim(\log n)^{2}$, for both time increments $\Delta t=1$ and $\Delta t=1 / n$ (see below). The parameters of the fitted curves agree well between model and data [Fig. 1(a); see the figure caption for details]. For comparison, the size dependence of depth in the AB model is also shown.

Going beyond averages and considering also fluctuations, a closer comparison between models and data is made in Fig. 2 by the Kolmogorov-Smirnov (KS) statistic. For a set of real trees $\mathcal{T}$, the cumulative depth distribution $q(d)$ is the fraction of trees in $\mathcal{T}$ having depth less than $d$. For each tree in $\mathcal{T}$, we generate with the rules of the model being tested 100 trees of the same size, obtaining a collection $\mathcal{T}^{\prime}$ of $100 \times|\mathcal{T}|$ model trees having the same size distribution as $\mathcal{T}$ and a cumulative depth distribution $q^{\prime}(d)$. The KS statistic is the maximum deviation $\kappa=\max _{d \in \mathbb{R}}\left|q(d)-q^{\prime}(d)\right|$ between data and model distributions, with $\kappa=0$ if and only if the distributions are identical. Except for the smallest trees $(n<20)$, we find (Fig. 2) that the depth distributions of the real trees in both databases are systematically closer to the age model with $\alpha=1.0$ than to the AB model.

\section{B. Analytic calculations for the age model and finite-size corrections}

At time step $n$ (and taking $\Delta t=1$ ), the tree has $n$ tips with ages $\tau_{1}=n, \quad \tau_{2}=n-1, \ldots, \tau_{i}=n-i+1, \ldots, \tau_{n}=1$. Thus the normalization constant is

$$
c_{\alpha}(n)=\sum_{i=1}^{n} \frac{1}{\tau_{i}^{\alpha}}=\sum_{k=1}^{n} \frac{1}{k^{\alpha}} .
$$

The asymptotic behavior of $c_{\alpha}(n)$ for large $n$ is

$$
c_{\alpha}(n) \sim \begin{cases}\frac{n^{1-\alpha}}{1-\alpha}, & \text { if } \alpha<1 \\ \log n, & \text { if } \alpha=1, \text { as } n \rightarrow \infty . \\ \zeta(\alpha), & \text { if } \alpha>1\end{cases}
$$

$\zeta(\alpha)$ is Riemann's zeta function, which is finite for $\alpha>1$. The expected age of the tip chosen at time $n$ is

$$
\overline{\tau(n)}=\frac{\sum_{\tau=1}^{n} \tau^{1-\alpha}}{c_{\alpha}(n)} \underset{n \rightarrow \infty}{\sim}\left\{\begin{aligned}
\frac{1-\alpha}{2-\alpha} n, & \text { if } \alpha<1 \\
\frac{n}{\log n}, & \text { if } \alpha=1 \\
\frac{n^{2-\alpha}}{\zeta(\alpha)(2-\alpha)}, & \text { if } 1<\alpha<2 \\
\frac{6}{\pi^{2}} \log n, & \text { if } \alpha=2 \\
\frac{\zeta(\alpha-1)}{\zeta(\alpha)}, & \text { if } 2<\alpha
\end{aligned}\right.
$$

This shows that the chosen age becomes progressively younger as $\alpha$ increases. Older branches become less likely to branch and imbalance is enhanced. Note that $\overline{\tau(n)} \sim n / 2$ for the ERM model $(\alpha=0)$.

A heuristic argument to obtain $\langle d(n)\rangle$ uses $\Delta n / \overline{\tau(n)}$ as an estimate of the mean number of branching events in a time interval of length $\Delta n$ centered at $n$. Thus we can count the mean number of branching events as a function of $n$ by the integral:

$$
\langle d(n)\rangle \approx \int_{1}^{n} d n^{\prime} \frac{1}{\overline{\tau\left(n^{\prime}\right)}} \underset{n \rightarrow \infty}{\sim}\left\{\begin{aligned}
\log n, & \text { if } \alpha<1 \\
(\log n)^{2}, & \text { if } \alpha=1 \\
n^{\alpha-1}, & \text { if } 1<\alpha<2 . \\
\operatorname{li}(n) \sim \frac{n}{\log n}, & \text { if } \alpha=2 \\
n, & \text { if } 2<\alpha
\end{aligned}\right.
$$

Prefactors have not been included since our crude argument is not expected to give them exactly. $\operatorname{li}(n)$ is the logarithmic integral function.

Equation (7) gives the $n$ dependence of the depth $\langle d\rangle$ in leading order for large tree size $n$. Finite size corrections to scaling, found numerically, are significant only close to the transition. Figure 3 shows finite size corrections to this leading order. As usual, the corrections become large close to a transition point, in this case close to $\alpha=1$ [Fig. 3(a)]. Likewise, $\alpha$ above but close to 1.0 leads to large corrections from the scaling with $n^{\alpha-1}$ [Fig. 3(c)]. For $\alpha=1.0$, the function $\langle d\rangle /(\ln n)^{2}$ is only weakly dependent on $n$, falling monotonically from 0.85 to 0.48 over 5 orders of magnitude in $n$ [filled triangles in Fig. 3(b)].

The predicted behavior (7) is consistent with our numerical findings. Thus the age model with $\alpha=1$ leads to the asymptotic square-logarithmic scaling that appears to describe the real phylogenies rather well. Interestingly, this particular scaling appears at the critical transition between 

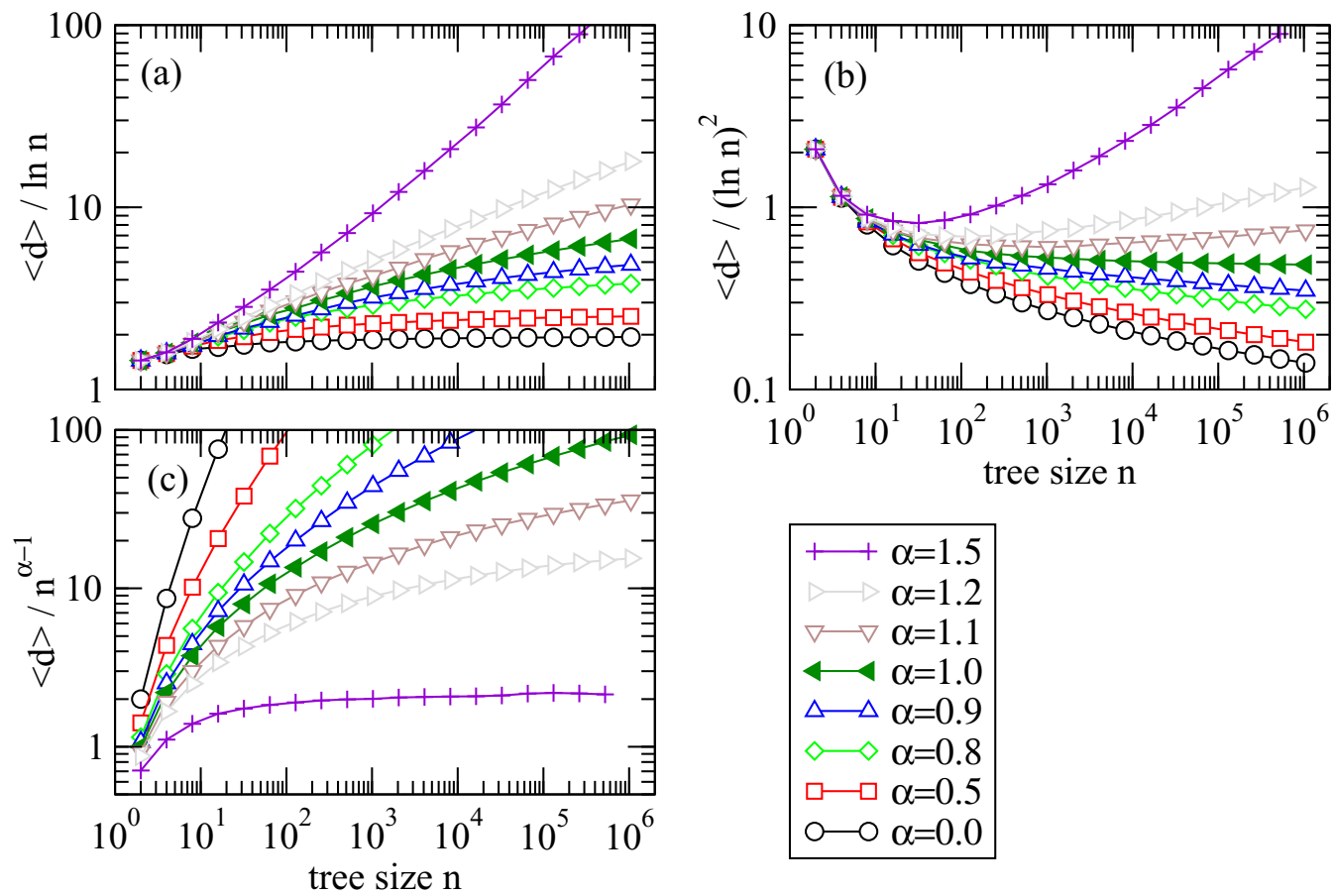

FIG. 3. (Color online) Analysis of finite size corrections. We plot the average depth of trees from the age model for various parameter values $\alpha$. Different panels use different rescaling of depth; see the $y$ axis labels. If finite size corrections from the scaling of Eq. (7) were absent, the three lower curves $(\alpha<1.0)$ would be flat in panel (a). In panel (b) this would be the case exactly for $\alpha=1.0$ (filled triangles). In panel (c), the three curves for $\alpha>1.0$ would be flat. Each data point is an average over 100 independent trees.

purely logarithmic and power-law scaling, in much the same way as for the beta-splitting model [31]. This may indicate a kind of universality in tree-shape transitions, and associated universality classes. Another transition, to the comb tree scaling, occurs at $\alpha=2$.

\section{Extensions}

The definition of the age model so far describes peripatric speciation. A more symmetric allopatric speciation mechanism would imply a more similar role for the species arising in a branching event, for example a resetting to zero of the age of the two species emerging from the branching, so that both are considered to be new and not just one of them. The analytic evaluations become more delicate, since $c_{\alpha}(t)$ is now a random variable, but heuristics confirms that the asymptotic scaling of the expected age $\overline{\tau(n)}$ and depth scaling $\langle d(n)\rangle$ in the symmetric model is the same as for the corresponding asymmetric one given by Eqs. (6) and (7). Numerically we find the mean depth obtained from the allopatric version to coincide with that of the original peripatric version of the model at $\alpha=1$ : Relative deviations between $\langle d\rangle$ estimates are below $1 \%$ and become smaller for growing $n$.

Another important extension corresponds to the case in which the age of the tips is not measured in number of speciation events $\tau$, but in a different but related time unit. It is biologically reasonable (as assumed also in the Yule model) that speciation rate is proportional to the number of species present, so that the instants of times assigned to successive speciation events $t_{n}$ and $t_{n+1}$ are related to the numbering of speciation events $n$ and $n+1$ by $\Delta t \equiv t_{n+1}-t_{n}=1 / n$. This implies $t_{n} \sim \log n$ at large $n$. This new time age $a_{n}(\tau)$ of a tip that has an event age $\tau$ is thus $a_{n}(\tau)=t_{n}-t_{n-\tau} \sim \log n-$ $\log (n-\tau)=-\log (1-\tau / n)$ for large $n$. For a version of the age model with speciation probabilities proportional to $1 / a_{n}^{\alpha}$, we can recalculate the expected value of the event age $\overline{\tau(n)}$ chosen at instant $n$ or $t_{n}$ :

$$
\overline{\tau(n)}=\frac{\sum_{\tau=1}^{n} \tau a_{n}(\tau)^{-\alpha}}{\sum_{\tau=1}^{n} a_{n}(\tau)^{-\alpha}} .
$$

To further analyze this expression, we approximate the sums by integrals, and introduce the change of variable $s=\tau / n$. After this it is clear that the integrals for large $n$ are dominated by the singularities arising as $s \rightarrow 0$ (say, within the interval $s \in[1 / n, \epsilon]$, with $\epsilon$ small), which allow us to use the small $s$ expansion, $a_{n}(\tau) \approx-\log (1-s) \approx s$ :

$$
\overline{\tau(n)} \approx \frac{\int_{\frac{1}{n}}^{\epsilon} n s\left(s^{-\alpha}+\cdots\right) d s}{\int_{\frac{1}{n}}^{\epsilon}\left(s^{-\alpha}+\cdots\right) d s} .
$$

Now, these integrals become identical to the ones corresponding to the asymptotic evaluation of the sums in the original age model, so the asymptotic behavior of the depth will be the same. This points out, once again, that the important ingredient needed to alter the standard logarithmic ERM depth scaling is the excess of branching probability assigned to young branches (small $\tau$ ) by the $\tau^{-\alpha}$ factor. Continuoustime branching processes in which different branches split independently with some waiting time distribution of the renewal type [18] can be also considered. They would have instantaneous branching rates decaying as $t^{-1}$ at long times when the waiting-time density decays as a power law at these 
long times. But as Eq. (9) reveals this long-time behavior is not the relevant one, but the presence of a short-time singularity. Singularity with the required strength can not be achieved with normalizable continuous-time waiting-time densities. This is why we have always found the normal depth scaling as $\log n$ in simulations of this type of processes, even when the waiting time distribution had fat tails. Another ingredient in the age model is that the normalization constant in the denominator of Eq. (3) involves the age of all branches. This provides an interaction among all branches, which is absent in models of independent branching.

\section{DISCUSSION}

Motivated by observations of anomalous scaling in evolutionary trees [11,12,21,22,24], the proposed age model introduces time correlations and branch interactions such that a variety of depth scalings can be reached beyond the standard logarithmic one. Remarkably, for the case $\alpha=1$, corresponding to the critical point between two qualitatively different phases, the model agrees with observed phylogenetic trees better than previous models. In addition, it describes the tree generation process assuming that lineages which have not speciated for a long time display in the future a still more reduced speciation rate. This kind of phenomenon is discussed in the framework of evolution and heritability of evolvability and robustness in the biological literature [34] and of phenotypic entrapment in genotype networks [35]. In a broader picture, dynamics on possibly rugged fitness landscapes [36] provides evolution at the microscopic level. It could serve as a mechanistic explanation of the assumptions of the age-dependent model.

Future work should consider the inclusion of extinction processes into the model. This is a realistic element that would open the possibility of an additional critical behavior (the transition between growth and extinction) known to alter tree topology [18,37-40]. Why evolution should be poised at the critical point deserves further investigation [41,42]. Analyses and comparison of branch length distributions are worth pursuing. Although branch length data of phylogenies are not as reliable as their topological structure [43], improvements are rapidly accumulating (see, e.g., Ref. [44]).

\section{ACKNOWLEDGMENTS}

We thank Alejandro Herrada, Stephan Steigele, and Joan Pons for valuable discussions regarding biological evolution. This work has been supported by MINECO (Spain) and FEDER (EU) through projects INTENSE@COSYP (FIS2012-30634) and MODASS (FIS2011-24785), and by Volkswagen Foundation through contract I / 82719.
[1] I. Rodriguez-Iturbe and A. Rinaldo, Fractal River Basins: Chance and Self-Organization (Cambridge University Press, Cambridge, 1997).

[2] G. B. West, J. H. Brown, and B. J. Enquist, Science 276, 122 (1997).

[3] T. Halpin-Healy and Y.-C. Zhang, Phys. Rep. 254, 215 (1995).

[4] E. Brunet, B. Derrida, and D. Simon, Phys. Rev. E 78, 061102 (2008).

[5] K. Klemm, V. M. Eguíluz, and M. San Miguel, Phys. Rev. Lett. 95, 128701 (2005).

[6] K. Klemm, V. M. Eguíluz, and M. San Miguel, Physica D 224, 149 (2006).

[7] M. M. Geipel, C. J. Tessone, and F. Schweitzer, Eur. Phys. J. B 71, 641 (2009).

[8] R. Gray and Q. Atkinson, Nature (London) 426, 435 (2003).

[9] B. Derrida, S. C. Manrubia, and D. H. Zanette, Phys. Rev. Lett. 82, 1987 (1999).

[10] M. Serva, J. Stat. Mech. (2005) P07011.

[11] E. A. Herrada, C. J. Tessone, K. Klemm, V. M. Eguíluz, E. Hernández-García, and C. M. Duarte, PLoS ONE 3, e2757 (2008).

[12] A. Herrada, V. M. Eguíluz, E. Hernández-García, and C. M. Duarte, BMC Evol. Biol. 11, 155 (2011).

[13] E. Brunet and B. Derrida, J. Stat. Mech. (2013) P01006.

[14] V. Sood, M. Mathieu, A. Shreim, P. Grassberger, and M. Paczuski, Phys. Rev. Lett. 105, 178701 (2010).

[15] J. Felsenstein, Inferring Phylogenies (Sinauer Associates, Sunderland, MA, 2003).
[16] M. J. Sanderson, M. J. Donoghue, W. Piel, and T. Eriksson, Am. J. Bot. 81, 183 (1994), data downloaded on June 30, 2007, http://www.treebase.org.

[17] S. Whelan, P. I. W. de Bakker, E. Quevillon, N. Rodriguez, and N. Goldman, Nucleic Acids Res. 34, D327 (2006), data downloaded on May 27, 2008, http://www.ebi.ac.uk/goldmansrv/pandit/.

[18] T. E. Harris, The Theory of Branching Processes (SpringerVerlag, Berlin, and Prentice-Hall, Englewood Cliffs, NJ, 1963).

[19] M. Simkin and V. Roychowdhury, Phys. Rep. 502, 1 (2011).

[20] I. Pinelis, Proc. R. Soc. London B 270, 1425 (2003).

[21] M. G. Blum and O. François, Syst. Biol. 55, 685 (2006).

[22] G. R. Jones, Syst. Biol. 60, 735 (2011).

[23] G. U. Yule, Philos. Trans. R. Soc. London B 213, 21 (1925).

[24] S. Pompei, V. Loreto, and F. Tria, PLoS ONE 7, e44849 (2012).

[25] A. Mooers and S. B. Heard, Q. Rev. Biol. 72, 31 (1997).

[26] F. A. Matsen, Syst. Biol. 55, 652 (2006).

[27] P. M. Agapow and A. Purvis, Syst. Biol. 51, 866 (2002).

[28] M. Sackin, Syst. Zool. 21, 225 (1972).

[29] M. Stich and S. C. Manrubia, Eur. Phys. J. B 70, 583 (2009).

[30] S. Keller-Schmidt and K. Klemm, Adv. Complex Syst. 15, 1250043 (2012).

[31] D. Aldous, in Random Discrete Structures, edited by D. Aldous and R. Pemantle (Springer, New York, 1996), pp. 1-18.

[32] D. Aldous, Stat. Sci. 16, 23 (2001).

[33] D. J. Ford, Ph.D. thesis, Stanford University, 2006, arXiv:math/0511246v1 [math.PR].

[34] J. Masel and M. L. Siegal, Trends Genet. 25, 395 (2009). 
[35] S. Manrubia and J. A. Cuesta, J. R. Soc. Interface 12, 20141010 (2014).

[36] P. Sibani, M. R. Schmidt, and P. Alstrøm, Phys. Rev. Lett. 75, 2055 (1995).

[37] M. Newman and P. Sibani, Proc. R. Soc. London B 266, 1593 (1999).

[38] P. De Los Rios, Europhys. Lett. 56, 898 (2001).

[39] E. Hernández-García, M. Tuğrul, E. A. Herrada, V. M. Eguíluz, and K. Klemm, Int. J. Bif. Chaos 20, 805 (2010).
[40] N. Becker and P. Sibani, Europhys. Lett. 105, 18005 (2014).

[41] P. Bak and K. Sneppen, Phys. Rev. Lett. 71, 4083 (1993).

[42] R. V. Solé and S. C. Manrubia, Phys. Rev. E 54, R42 (1996).

[43] T. Barraclough and S. Nee, Trends Ecol. Evol. 16, 391 (2001).

[44] C. Venditti, A. Meade, and M. Pagel, Nature (London) 463, 349 (2010). 